\title{
Correlation between aromatase expression in the eutopic endometrium of symptomatic patients and the presence of endometriosis
}

This article was published in the following Dove Press journal:

International Journal of Women's Health

22 February 2012

Number of times this article has been viewed

\author{
Hugo Maia Jr ${ }^{1,2}$ \\ Clarice Haddad ${ }^{1,2}$ \\ Julio Casoy' \\ 'CEPARH, ${ }^{2}$ taigara Memorial Day \\ Hospital, Salvador, Bahia, Brazil
}

Correspondence: Hugo Maia Jr Centro de Pesquisa e Assistência em Reprodução Humana (CEPARH), Rua Caetano Moura, 35, 402 I0-34I

Salvador, Bahia, Brazil

Tel +55 7| 32478216

Fax $+55713235-3442$

Email ceparh@uol.com.br
Objective: To investigate whether aromatase expression in the eutopic endometrium correlates with the presence and severity of endometriosis in patients with infertility and/or dysmenorrhea undergoing laparoscopy and hysteroscopy.

Patients: The study involved 106 patients of reproductive age with symptoms of dysmenorrhea and infertility. Sixteen endometriosis-free asymptomatic patients were used as a control group.

Methods: Concomitant laparoscopy and hysteroscopy was carried out in all cases. An endometrial biopsy was taken to determine aromatase $\mathrm{p} 450$ expression by immunohistochemistry. Endometriosis was staged according to the American Society of Reproductive Medicine classification.

Results: Endometriosis was diagnosed by laparoscopy in 92/106 symptomatic patients. In this group, aromatase expression was detected in the eutopic endometrium of 66/92 patients with endometriosis (72\%) and in 13/14 (95\%) patients in the symptomatic, endometriosis-free group $(P=0.09)$. Aromatase expression was not detected in any patients from the control group. In the endometriosis group, aromatase expression was detected in the eutopic endometrium of 28/45 patients (62\%) with American Society of Reproductive Medicine classification stage 1 of the disease, in $11 / 14$ patients $(78 \%)$ with stage II, $14 / 20$ patients $(70 \%)$ with stage III, and in $12 / 13$ patients $(92 \%)$ with stage IV; however, the difference was only statistically significant between stages I and IV $(P=0.04)$.

Conclusion: Aromatase expression in the endometrium was associated with the presence of dysmenorrhea and infertility irrespective of the presence of endometriosis. When endometriosis was present, however, there was a tendency for aromatase expression to be positively correlated with dysmenorrhea severity.

Keywords: aromatase, endometrium, endometriosis, Cox-2, dysmenorrhea

\section{Introduction}

The onset of clinical symptoms associated with endometriosis may predate clinical diagnosis by many years. ${ }^{1}$ Although this may simply reflect a lack of sensitive, noninvasive methods capable of diagnosing this pathology at an early stage, it is also possible that the enzymatic and inflammatory changes that signal the development of endometriosis may be initiated in the endometrium before the disease is established in the pelvis. These functional changes may involve the upregulation of enzymes related to estrogen synthesis that are overexpressed in the eutopic endometrium of patients with endometriosis, where they may play a pivotal role not only in the implantation failure associated with this pathology but also in the onset of menstrual symptoms. ${ }^{2-4}$ 
It is known that both endometriotic lesions and the eutopic endometrium are able to express aromatase, a key enzyme in the transformation of androgens into estrogens. The release of these enzymes is stimulated by inflammatory mediators such as the prostaglandins produced by the action of cyclooxygenase-2 (Cox-2). ${ }^{2,3}$ The aggressiveness of endometriosis appears to correlate with the intensity of the expression of this enzyme in lesions, with the most aggressive occurring in red peritoneal lesions. ${ }^{2}$ A correlation was also found between the severity of endometriosis and the intensity of Cox-2 expression in both lesions and the eutopic endometrium, thus suggesting a role of locally produced estrogens in the upregulation of this enzyme. ${ }^{3-5}$

Nevertheless, the role played by aromatase and Cox-2 expression in the eutopic endometrium in relation to the development of endometriosis and in the intensity of menstrual-related symptoms is not totally understood. It was recently suggested that endometriosis may commence as an endometrial disease provoked by the aberrant expression of aromatase in this tissue, as this appears to play a pivotal role in its survival in ectopic locations. ${ }^{4}$ Gene array studies have shown that the expression profiles of endometriosis lesions and the eutopic endometrium are similar, which supports the hypothesis that endometriosis is an endometrial disease. ${ }^{6}$ It is biologically plausible that the presence of aromatase activity in the endometrium is pivotal for the development of endometriosis by blocking phagocytosis by activated macrophages and may also be responsible for the exacerbation of menstrual symptoms whose onset predates the diagnosis of this pathology. ${ }^{1,4,7}$ In this hypothesis, endometriosis would be a late consequence of enzymatic changes that occur in the endometrium prior to its establishment outside the uterus, which could explain the lag that is often reported between the onset of symptoms and diagnosis of the disease.

This hypothesis could be tested by examining patients with severe dysmenorrhea or infertility to determine whether laparoscopy reveals endometriosis despite aromatase expression already being positive in the endometrium. In the present study, aromatase expression was determined in the eutopic endometrium of patients with severe menstrual symptoms and/or infertility who underwent laparoscopy and hysteroscopy as a part of their diagnostic work-up.

\section{Methods}

A total of 106 patients of reproductive age (range $=18-47$ years) received a laparoscopy and hysteroscopy with endometrial biopsy that was carried out using a $4 \mathrm{~mm}$ Karman curette attached to a $10 \mathrm{~mL}$ syringe to produce a vacuum, which is the standard procedure for the evaluation of menorrhagia, severe dysmenorrhea, or infertility in the day hospital where the current study was carried out. All patients included in this group had reported at least two of these three symptoms for periods ranging from 2 to 8 years. The control group consisted of sixteen patients who received a laparoscopy for reasons unrelated to infertility or severe dysmenorrhea and who were found to be endometriosis-free during the examination. The indications for laparoscopy in this group were either benign non-endometriotic ovarian cysts (seven patients) or tubal ligation (nine patients). In these patients, an endometrial biopsy was performed at the time of the laparoscopy using a $4 \mathrm{~mm}$ Karman curette.

The patients enrolled in the study gave written informed consent for immunohistochemical studies to be carried out on the endometrial biopsy specimens obtained as part of their standard medical care. This study was approved by the institution's internal review board.

Laparoscopies were performed either during the proliferative phase $(\mathrm{n}=72)$ or in the secretory or menstrual phase ( $n=34$ ), since the timing of the procedure depended on the availability of the surgical theater and the specifics of the institution's waiting list. The laparoscopy reports and color images were retained in the patients' files for further evaluation if necessary. During the laparoscopy procedure, the surgical team ascertained the presence of endometriosis and graded the stage of the disease in accordance with the American Society of Reproductive Medicine (ASRM) classification. ${ }^{8}$ Using laparoscopic classification criteria, the patients with endometriosis were divided into four groups according to the severity of their lesions, using the ASRM classification of minimal (stage I), mild (stage II), moderate (stage III), or severe (stage IV). The severity of menstrual symptoms such as dysmenorrhea, the presence or absence of infertility, and the reason for laparoscopy were recorded in the patients' files.

Endometrial samples were fixed in $10 \%$ formalin before being assessed. Immunohistochemistry was performed following antigen retrieval to detect the presence of aromatase p450. Aromatase expression was investigated using the commercially available monoclonal antibody MCA2077 clone H4 (Serotech, Raleigh, NC). Antigen retrieval was performed using Tris-EDTA buffer (pH 8.0; Sigma-Aldrich, São Paulo, Brazil). The reaction was revealed using the streptavidin-biotin method. The presence of aromatase expression was rated as positive if there was any detectable staining reaction in the glandular epithelium or negative when no reaction was observed. Placental tissue and an atrophic endometrial sample were used as positive 
and negative controls, respectively, in all immunostaining reactions for aromatase $\mathrm{p} 450$. Statistical analysis was performed using the one-tailed chi-square test for differences in percentages, using an unnamed online interactive calculation tool for chi-square tests of goodness of fit and independence, which is hosted at http://quantpsy.org. Results were considered statistically significant at $P<0.05$.

\section{Results}

In the group of patients with severe dysmenorrhea, endometriosis was diagnosed in 90/106 patients. In the remaining 16 cases, the pelvis was found to be normal during the laparoscopy.

Aromatase expression in the eutopic endometrium was detected by immunohistochemistry mainly in the glandular epithelium (Figure 1). The staining reaction was positive in 66/92 patients $(72 \%)$ with endometriosis and symptoms of dysmenorrhea and/or infertility, and there were no significant differences between the phases of the patient's menstrual cycle. In symptomatic patients who were found to have normal pelvic results during the laparoscopy, aromatase expression was still positive in the endometrium in $13 / 14$ cases (95\%), though this difference was not statistically significant $(P=0.09)$. In the asymptomatic control group without endometriosis, however, aromatase expression was negative in the eutopic endometrium in all cases.

In the subset of symptomatic patients with a laparoscopic diagnosis of endometriosis, the percentage of endometrial samples positively expressing aromatase in the glandular epithelium showed a trend toward greater positivity as the severity of the disease increased in accordance with the ASRM criteria. Aromatase expression was detected in the eutopic endometrium of $28 / 45$ patients with stage I

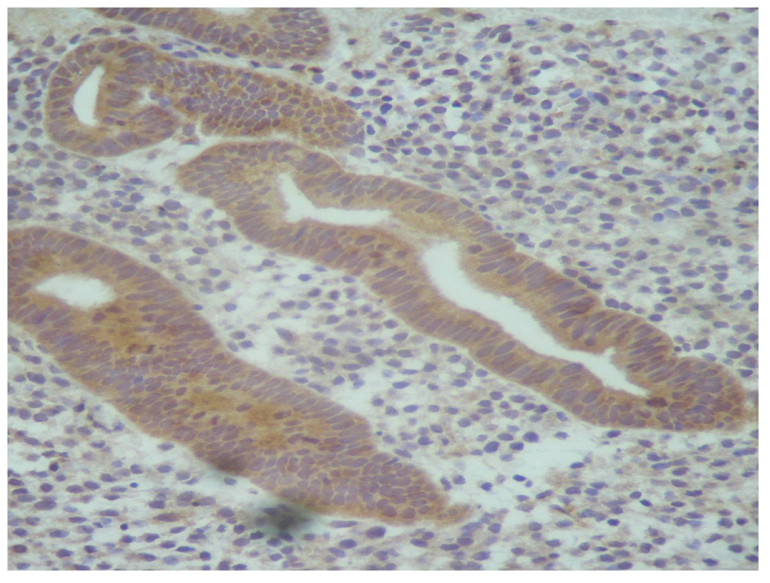

Figure I Aromatase expression in the eutopic endometrium detected by immunohistochemistry. endometriosis (62\%), 11/14 patients with stage II (78\%), $14 / 20$ patients with stage III (70\%) and 12/13 patients with stage IV endometriosis (92\%). However, only the difference between stages I and IV of the disease was statistically significant $(P=0.04)$ (Table 1$)$.

\section{Discussion}

The present study not only showed an association between the presence of aromatase expression in the eutopic endometrium and the severity of endometriosis lesions at laparoscopy but also suggests that this expression was already present in symptomatic patients in whom pelvic endometriosis was not found. These findings agree with the hypothesis that endometriosis may commence as an endometrial disease resulting from functional changes in the endometrium before the disease establishes outside the uterus.

The presence of similar epigenetic changes in the eutopic endometrium and the endometriotic lesions further corroborates the hypothesis that endometriosis is an endometrial disease. ${ }^{6}$ The positive correlation between mRNA levels in the eutopic endometrium and the severity of endometriosis and adenomyosis also supports this hypothesis. ${ }^{9}$ Whether endometriosis starts as an endometrial disease before establishing itself elsewhere in the pelvis ${ }^{6,7}$ cannot be proven by the present study, but previous work shows that the onset of symptoms associated with this pathology may commence many years prior to its diagnosis, which support this hypothesis. ${ }^{1}$ The occurrence of severe dysmenorrhea for many years prior to a diagnosis of endometriosis may reflect these initial endometrial changes favoring the upregulation of both estradiol and prostaglandin production in this tissue. ${ }^{4,6,7}$ The occurrence of such symptoms in patients with a normal pelvis at laparoscopy, as shown in the present study, may suggest that these functional changes in the endometrium are not only associated with the onset of endometriosis-related symptoms but that they may occur prior to the diagnosis of any pelvic pathology by laparoscopy. Functional endometrial changes leading to an increase in aromatase expression

Table I Aromatase expression in the eutopic endometrium and the laparoscopic classification of endometriotic lesions according to American Society of Reproductive Medicine classification

\begin{tabular}{ll}
\hline Type of lesion & Aromatase positive \\
\hline Stage I & $28 / 45(62 \%)$ \\
Stage II & $11 / 14(78 \%)$ \\
Stage III & $14 / 20(70 \%)$ \\
Stage IV & $12 / 13(92 \%)$ \\
\hline
\end{tabular}

Notes: Chi-square test: stages I and IV, $P<0.05$. The other differences were not statistically significant. 
may therefore predate the development of endometriosis, initially provoking dysmenorrhea and possibly infertility before triggering the development of the disease outside the uterus. ${ }^{4}$ It still remains to be determined whether this group of symptomatic patients represents a high-risk population for the development of endometriosis. In patients with an established diagnosis of endometriosis, however, endometrial positivity for aromatase tends to increase with the severity of the disease. This may be due to the greater possibility of spontaneous regression of early stage endometriosis, which may occur because of reduced aromatase activity in the eutopic endometrium.

Although aromatase expression in the eutopic endometrium may be pivotal for the onset and progression of endometriosis, it has yet to be clarified how this enzyme affects the development of endometriosis. The role played by estrogens in inhibiting immune surveillance by activated macrophages has been suggested as one of the likely mechanisms by which aromatase affects the course of endometriosis., ${ }^{4,10}$ This local estrogen production in the endometrial cells shed to the pelvis in retrograde menstruation may favor their survival in these ectopic locations by directly preventing their destruction by activated immune cells through the inhibition of the phagocytosis mechanism. ${ }^{10-13}$

The presence of aromatase in the eutopic endometrium may therefore represent the initial step in the development of endometriosis and could be necessary for the development of lesions outside the uterus. ${ }^{7}$ The similar genetic alterations found between endometriotic lesions and the respective eutopic endometrium also indicate a common histological origin. ${ }^{7}$

The current study's finding that aromatase expression is detectable by immunohistochemistry in the endometrium of symptomatic, endometriosis-free patients agrees with the hypothesis that this enzyme initially exacerbates dysmenorrhea or causes infertility prior to facilitating the development of endometriosis elsewhere in the pelvis. Nevertheless, a temporal relationship cannot be established solely from these data. A previous study has shown that aromatase expression in the endometrium reduces implantation rates in patients undergoing in vitro fertilization, however, which may help to explain the lower fertility rate of women with endometriosis. ${ }^{14}$

The presence of aromatase and other enzymes related to estrogen metabolism in the eutopic endometrium will ultimately create a hyperestrogenic milieu, resulting in increased prostaglandin production, particularly because Cox- 2 expression is upregulated by estradiol in this tissue..$^{6-9,12,13}$ This would explain why the pelvis of a patient with severe dysmenorrhea or infertility may be normal, as was reported in the current study - aromatase expression in the eutopic endometrium may be sufficient to provoke these symptoms by enhancing the production of proinflammatory prostaglandins in this tissue. However, the possibility that some of these endometriosis-free patients have adenomyosis cannot be completely excluded in this study, despite the fact that myometrial findings were normal at transvaginal sonography.

Estrogens produced locally in the endometriotic lesions and eutopic endometrium result in the mechanism of decreased immune surveillance and enhanced tolerance found in endometriosis, ${ }^{14}$ which may explain the inverse relationship between estrogen levels and impaired NK-cell function found as a function of the severity of the disease. ${ }^{15}$ Local estrogen production would also stimulate the production of VEGF, growth factors, and inflammatory cytokines by immune cells, thus favoring the progression of endometriosis. ${ }^{12,15,16}$ The blockade of phagocytosis by the local production of estrogens and the concomitant stimulation of growth and inflammatory factors would explain the paradoxical effects of macrophages in endometriosis, which seem to interact with rather than destroy these ectopic endometrial cells, thus enhancing their growth and angiogenesis. ${ }^{12,17}$

In conclusion, a vicious cycle of enhanced inflammation and local estrogen production mediated by Cox- 2 and aromatase expression in the eutopic endometrium of endometriosis patients may play a pivotal role not only in determining the clinical course of this disease but also in defining the intensity of its debilitating symptoms. In the pelvic cavity, the ensuing inflammatory reaction provoked by the arrival of endometrial cells will further exacerbate their preexisting aromatase activity, thus augmenting local estrogen production and halting phagocytosis by activated macrophages. ${ }^{6,7}$

\section{Acknowledgments}

The authors are grateful to Dr Nathanial Pinheiro for his skilled assistance in interpreting the pathology slides.

\section{Disclosure}

The authors declare that they received no financial support for this study.

\section{References}

1. Hadfield R, Mardon H, Barlow D, Kennedy S. Delay in the diagnosis of endometriosis: a survey of women from the USA and the UK. Hum Reprod. 1996;11(4):878-880.

2. Bukulmez O, Hardy DB, Carr, BR, Word RA, Mendelson CR. Inflammatory status influences aromatase and steroid receptor expression in endometriosis. Endocrinology. 2008;149(3):1190-1204. 
3. Cho S, Park SH, Choi YS, et al. Expression of cyclooxygenase-2 in eutopic endometrium and ovarian endometriotic tissue in women with severe endometriosis. Gynecol Obstet Invest. 2010;69(2):93-100.

4. Maia H Jr, Casoy J, Valente Filho J. Is aromatase expression in the endometrium the cause of endometriosis and related infertility? Gynecol Endocrinol. 2009;25(4):253-257.

5. Buchweitz O, Staebler A, Wülfing P, Hauzman E, Greb R, Kiesel L. COX-2 overexpression in peritoneal lesions is correlated with nonmenstrual chronic pelvic pain. Eur J Obstet Gynecol Reprod Biol. 2006; 124(2):216-221.

6. Borghese B, Barbaux S, Mondon F, et al. Research resource: genome-wide profiling of methylated promoters in endometriosis reveals a subtelomeric location of hypermethylation. Mol Endocrinol. 2010;24(9):1872-1885.

7. Maia H Jr, Casoy J, Correia T, Freitas LA, Pimentel K, Athayde C. The effect of oral contraceptives on aromatase expression in the eutopic endometrium of patients with endometriosis. Gyneco Endocrinol. 2008; 24(3):123-128.

8. American Society for Reproductive Medicine. Revised ASRM classification for endometriosis: 1996. Fertil Steril. 1997;67:817-821.

9. Hatok J, Zubor P, Galo S, et al. Endometrial aromatase mRNA as a possible screening tool for advanced endometriosis and adenomyosis. Gynecol Endocrinol. 2010;27(5):331-336.

10. Cakmak H, Guzeloglu-Kayisli O, Kayisli UA, Arici A. Immune-endocrine interactions in endometriosis. Front Biosci (Elite Ed). 2009;1:429-443.
11. Provinciali M, Di Stefano G, Muzzioli M, Garzetti GG, Ciavattini A, Fabris N. Relationship between 17-beta-estradiol and prolactin in the regulation of natural killer cell activity during progression of endometriosis. J Endocrinol Invest. 1995;18(8):645-652.

12. Lin YJ, Lai MD, Lei HY, Wing LY. Neutrophils and macrophages promote angiogenesis in the early stage of endometriosis in a mouse model. Endocrinology. 2006;147(3):1278-1286.

13. Eyster KM, Hansen KA, Winterton E, Klinkova O, Drappeau D, Mark-Kappeler CJ. Reciprocal communication between endometrial stromal cells and macrophages. Reprod Sci. 2010;17(9):809-822.

14. Brosens J, Verhoeven H, Campo R, et al. High endometrial aromatase P450 mRNA expression is associated with poor IVF outcome. Hum Reprod. 2004;19(2):352-356.

15. Cakmak H, Guzeloglu-Kayisli O, Kayisli UA, Arici A. Immune-endocrine interactions in endometriosis. Front Biosci (Elite Ed). 2009;1:429-443.

16. Provinciali M, Di Stefano G, Muzzioli M, Garzetti GG, Ciavattini A, Fabris N. Relationship between 17-beta-estradiol and prolactin in the regulation of natural killer cell activity during progression of endometriosis. J Endocrinol Invest. 1995;18(8):645-652.

17. Eyster KM, Hansen KA, Winterton E, Klinkova O, Drappeau D, Mark-Kappeler CJ. Reciprocal communication between endometrial stromal cells and macrophages. Reprod Sci. 2010;17(9):809-822.
International Journal of Women's Health

\section{Publish your work in this journal}

The International Journal of Women's Health is an international, peerreviewed open-access journal publishing original research, reports, reviews and commentaries on all aspects of women's healthcare including gynecology, obstetrics, and breast cancer. Subject areas include: Chronic conditions (migraine headaches, arthritis, osteoporosis);

\section{Dovepress}

Endocrine and autoimmune syndromes; Sexual and reproductive health; Psychological and psychosocial conditions. The manuscript management system is completely online and includes a very quick and fair peer-review system. Visit http://www.dovepress.com/ testimonials.php to read real quotes from published authors. 